\title{
استراتيجية تعليم اللغة العربية في عصر العولمة
}

Rizal Firdaus, Kurnia Afandi Al Farisi, Robi Mandala

Sekolah Tinggi Ilmu Bahasa Arab Ar-Raayah Sukabumi

rizal@arraayah.ac.id, kurniaafandi98@gmail.com, robymandala123@gmail.com

\begin{tabular}{|l|l|l|} 
Received: November 17th 2019 & Revised: December $4^{\text {th }} 2019$ & Accepted: December 30th 2019
\end{tabular}

Abstract: Arabic is the only bigh language that has been valued to maintain its entity and become scientific. The book shows a correct and accurate understanding and taste only in Arabic, and the heritage books of Islamic sciences are written in that language. As time fluctuates like the ball, the situation has changed in times and in our present time human beings live in the era of globalization where western countries dominate all parts of the world and in all their fields such as the field of economy and education, the Islamic countries were affected by the entry of Western ideas, culture and traditions that lead to the weakness of the Arabic language. Therefore, teaching Arabic in the age of globalization requires a variety of and appropriate means, methods and methods, and this research aims to develop the appropriate methods and teaching strategy for teaching The Arabic language in the age of globalization, and one of the ways that can be used in the age of globalization are language games, which are designed for the purpose of Language learning, expanding or developing certain concepts to understand historical or cultural events or to help children acquire language skills through play. Language games are divided into multiple types, divided according to basic language skills, namely oral games and reading games. In this research, the authors will show about different language games that can be used in the teaching of Arabic language in the age of globalization depending on language skills, including: (1) character, word and sentence recognition games. (2) Reading training games from right to left. (3) Training games to read a word or phrase and short text. (4) Games to recognize and correct reading errors. (5) Vocabulary absorption training games, short phrases or short text.

Keyword: globalization, Arabic language education, language games. 
إن للغة أهمية بالغة في حياة الإنسان، وأنها من أهم الظواهر الاجتماعية التي أنتجها التطور البشري، حيث أنها تقوم بدور هام جدا في المجتمع، وهو باعتبارها أهم وسيلة من وسائل التواصل والتفاهم الأساسية بين البشر، أذ تسهم في بنا العلاقات الاجتماعية السليمة بين أفراد المجتمع الواحد من جهة وفيما بين المجتمعات المختلفة من جهة أخرى. بالإضافة لكونها تسهم في نقل الثقافة والتراث الإنساني من جيل إلى آخر، وفي تراكم المعارف والخبرات.

ولقد لعبت اللغة دورا هاما في تحقيق المنزلة العليا للإنسان بين الكائنات الأخرى، وهي على خلاف الأشكال الأخرى للحياة الإنسانية، قد تطورة بسرعة في فترات متلاحقة، وهي في تطورها تزود الأجيال الإنسانية بالأدوات الفعّالة للتقدم والتطور.وإن للغة خصائصها وقواعدها التي تميّزها عن غيرها، كاللغة العربية وهي إحدى اللغات التي لها خصائص ميّزة و مختلفة عن غيرها في كثير من مظاهر الصوت والدلالة والقواعد والمفردات.واللغة العربية هي اللغة السامية الوحيدة التي قدر لها تحافظ على كيا نها وأن تصبح علمية، وماكان ليتحقّق لها ذلك لولا نزول القرآن الكريم بها، إذلا يمكن فهم ذلك الكتاب المبين الفهم الصحيح والدقيق وتذوق إلاّ بلغة العربية، كما أن كتب التراث من العلوم الإسلامية مكتوب بتلك اللغة ،ومن هنا تعلّم العربية مطمحا لكلّ" المسلمين الذين يبلغ عددهم نو مليار مسلم في شتى أنخاء العالم.واللغة العربية بمفهومها المعاصر هي اللغة https://jurnal.yudharta.ac.id/v2/index.php/studi-arab 
الفصحى التى استخدمها العرب الجاهليون في أدبهم، وتلك اللغة التي نزل بها القرآن

$$
\text { الكريم، والتي ما زالت تستخدم حتى اليوم في المواقف الخطاب الرسمي. }
$$

وإن تعليم اللغة العربية لها أهمية بالغة ودور بارز ومهم في حفاظ على التراث

الإسلامي: القرآن الكريم والحديث الشريف.واللغة العربية ليست مادة لغوية فحسب

لكنها وسيلة لدراسة المواد الأخرى التي تتعلق بالعلوم الشرعية، مثل:الفقه والحديث

والتفسير والتوحيد وغير ذلك من العلوم الشرعية. وإن الزمان تتقلب كتقلب الكرة، فتغيرت الأحوال بتغير الأزمان وفي زماننا الحاضر يعيش الإنسان في عصر العولمة حيث أن بلاد الغربية يسيطر أنحاء العالم كلها وفي كل مجالاتها مثل مجال الاقتصاد والتعليم فتأثرت الدول الإسلامية بدخول الأفكار والثقافة والتقاليد الغربية التي تؤدي إلى ضعف اللغة العربية.والناس تختلفون في اتجاه العولمة منهم من يرى أن في العولمة دعوة إلى تقسيم العمل وانتشار التكنولوجيا الحديثة من مراكزها في العالم المتقدم اقتصاديا، إلى أقصى أطراف الأرض، ومن ثم زيادة الإنتاج.وهناك المفتونون بالمدنيّة الغربيّة بوجه عام، ليس بنتاجها المادّيّ فحسب، بل في مجال نقل المعلومات وتخزينها وتوفيرها، وبما حقّقه الغرب في المجال السّياسي والإجتماعي والثّقافي.وهناك أيضا الكارهون للعولمة،ولديهم أسباب لهذه الكرهية، فهناك من يكرهها لأن فيها مزيدا من الاستغلال الاقتصاديّ.وهناك من يكره العولمة لا بسبب اقتصاديّ، بل لسبب دينيّ، فالعولمة آتية من مراكز دينها غير ديننا، بل هي قد تنكرت للأديان كلّها، وآمنت بالعلمانية التي لا تختلف كثيرا، في نظر هؤلاء، عن الكفر. 
إن كلّ من المواقف المؤيدة والرافضة للعولمة، يحمل في رأي البعض جزءا من

الحقيقة.نعم، إن العولمة قد تؤدي إلى زيادة الإنتاج، والعولمة قد تمثل تقدما في بعض القدرات للإنسان، وفي بعض أنواع الإنتاج العلميّ والفنيّ. ولكن العولمة تتضمن، بلا شكّ، اتجاها نحو السيطرة الاقتصادية من الشركات الكبيرة للمستضعفين في الأرض. وهكذا تأثير العولمة في جوانب ومجالات الحياة الإنسانية، وفي مجال التعليم كذلك لا يخلو من تأثير

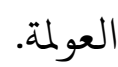

وهنا جاء دور مهم للقائمين في مجال التعليم أن يختاروا استراتيجية ووسائل وطرق

التي تناسب عصر العولمة ومن هنا يريد الكاتبان أن تقدما استراتيجية تعليم اللغة العربية التي تناسب عصر العولمة. مفهوم الاستراتيجية التعليم تعريف الاستراتيجية

إن مصطلح استراتيجية هو مصطلح الذى استخدم كثيرا في مجال العسكري وهو مصطلح عام بمعنى الطريقة أو الأسلوب.وطريقة تطلق عن تطبيق استراتيجيات وأخص من طريقة هي أسلوب، واستراتيجيات وطريقة وأسلوب هي مصطلاحات تستخدم للوصول إلى الهدف.والفرق بين الطريقة والأسلوب في التدريس، أما الطريقة هي خطط عامة لاختيار المادة وتنظيمها وعرضها، وأما الأسلوب هي الخطوات والإجراءات التى تتم في الصف للدرس معين. إذن المقصود بالطريقة التدريس الخطة الشاملة التي يستعين بها https://jurnal.yudharta.ac.id/v2/index.php/studi-arab 
المدرس، لتحقيق الأهداف المطلوبة من تعلم اللغة.وتتضمن الطريقة ما يتبعه المدرس من أساليب، وإجراءات، وما يستخدمه من مادة تعليمية، ووسائل معينة.أو الإجراءات التى يتبعها المعلم لمساعدة طلابه على تحقيق الأهداف، وقد تكون تلك الإجراءات مناقشات أو توجيه أسئلة أو تخطيطا لمشروع أو إثارة لمشكلة، أو تهيئة لموقف معين، يدعو طلابه إلى تساؤل أو محاولة الاكتشاف، أو فرض الفروض، أو غير ذلك من الإجراءات(أحمد حسين اللقانى،على أحمد الحجيلى:1999).أما الاستراتيجية هي مجموعة من قرارات يتخذها المعلم، وتنعكس تلك القرارات في أنماط من الأفعال يؤديها المعلم والتلامذ في المواقف التعليمي.والعلاقة بين الأهداف التعليمية والاستراتيجية المختارة علاقة جوهرية حيث يتم اختيار الاستراتيجية على أساس أنها أنسب وسيلة لتحقيق الأهداف. ب) تعريف تعليم

معنى التعليم في اللغة هي مصدر من (علم يعلّم تعليما) يطلق على عدة معاني منها: وضع علامة، يقال علّم الشيء أو علّم على الشيء، بمعنى وضع عليه علامة.كما نقول علّم مقطعا في الكتاب أو علّم على فقرة أي أسماء الغائبين.وعلّم عليه بعلامة في رقبته أو فهم أو درّبه.يقال: علّمه القراءة أي جعله يعرفها، فهمها إياها .وعلّمه الكتابة والرمايّة أي درّبها عليها، وعلّم الناشئة، وفي الروية "علموا أولادكم السباحة والرماية والفروسية"(من كلام عمر بن الخطاب)، وفي قوله تعالى: "وعلمك مالم تكن تعلم"(النساء:113)، وما علمتم من الجوارح " (المائدة : 40) أي وما دربتم (أحمد 
حسين اللقانى وعلى أحمد الحجيلي:1999) وفي الاصطلاح يعرف التعليم بأنه عملية منظمة تهدف إلى اكتساب الشخص المتعلم للأسس العامة الباينة للمعرفة، ويتم ذلك بطريقة منظمة ومقصودة وبأهداف محددة ومعروفة.ويمكن القول أن التعليم هو عبارة عن نقل للمعلومات بشكل منسق للطالب، أو أنه عبارة عن معلومات، ومعارف، وجبرات، ومهارات يتم اكتسابها من قبل المتلقي بطرق معينة (طارق محمود:2017).

والخلاصة أن معنى استراتيجية التعليم هو خطوات إجرائية منتظمة ومتسلسلة بحيث تكون شاملة ومرنة ومراعية لطبيعة المعلمين التى تمثل الواقع الحقيقي لما يحدث داخل قاعة الدراسة من استغلال الامكانات متاحة لتحقيق مخرجات تعليمية مرغوب فيها. مفهوم اللغة العربية معنى اللغة

لقد عرّفت العلماء اللغة تعريفات مختلفة، منها "بأنها تشير إلى مجموعة منظمة من العادات الصوتية التي يتفاعل بواسطتها أفراد المجتمع الإنساني https://jurnal.yudharta.ac.id/v2/index.php/studi-arab 
ويستخدمونها في أمور حياتهم".ووعرّف ابني جني اللغة بقوله ."حد اللغة أصوات يعبّر بها كل قوم عن أغراضهم". وأما اللغة بتعريف العام " هي مجموعة التعبيرات الإرادية (المقصودة ) التي يستخدمها الكائن الي( الإنسان ) بهدف الاتصال ببني جنسه".3 ب) خصائص اللغة من خصائص اللغة ما يلى: - من

1) اللغة سمة انسانية، أى خاصة بالإنسان وحده، وهي على هذا يجب أن تكون دائما في خدمة أهدافه وأفراده الحقيقة، وأن ترتبط بنموه إجتماعيا أو إقتصاديا وفكريا. 2) اللغة صوتية، وهذه الخاصية تعني أن الطبيعة الصوتية لغة هي الأساس، بينما يجيء الشكل المكتوب لها في المرتبة التالية من حيث الوجود.

3) واللغة تحمل المعنى، ومعنى هذه الخاصية أن اللغة تتكون من رموز لها معان وهذه الرموز يعرفها كل من المتكلم والسامع، والكاتب، والقارئ، وبدون هذه المعرفة الثابتة للمعانى يصبح الاتصال صعبا إن لم يكن مستحيلا، وينبنى أن يكون واضحا أن صلة بين الرمز والثيء الذى يعنيه صلة عرفية، أي ليست طبيعية. 4) واللغة ذات نظام خاص، وتعنى هذه الخاصية أن أي لغة تتكون من وحدات خاصة، وهذه وحدات تحدث في أنماط ثابتة.

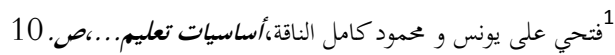

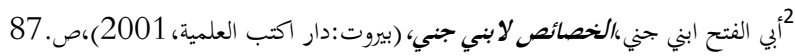

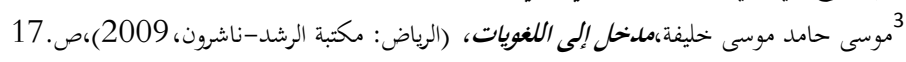

Program Studi Pendidikan Bahasa Arab Universitas Yudharta Pasuruan https://jurnal.yudharta.ac.id/v2/index.php/studi-arab 
5) واللغة سلوك مكتسب، ومعنى هذا أن العادات اللغوية المختلفة يكتسبها الفرد

$$
\text { من المجتمع الذي يعيش فيه. }
$$

6) اللغة نامية، أي أن اللغة في حالة تغير دائم، ويمكن ملاحظة هذا التغير في أنظمة

$$
\text { الأصوات، والقواعد والمفردات من جيل إلى جيل. }
$$

ومن وظائف اللغة،ما يلى:

1) اللغة وظيفتها التعبير عن الأفكار والعواطف والانفعالات

2) اللغة وظيفتها تصريف شؤون المجتمع الإنساني.

من هنا وجدنا البعض يفصل الوظيفتين السابقتين للغة فيما يلي من وظائف:

$$
\begin{aligned}
& \text { 1) تنسيق الأنشطة بين أعضاء المجتمع. } \\
& \text { 2) تثبيت الفكر والتعبير عنه. } \\
& \text { 3) إيصال الأفكار والمشاعر. } \\
& \text { 4) تمتيح النفس. } \\
& \text { 5) تقليل الاضطراب. } \\
& \text { د) اللغة العربية }
\end{aligned}
$$

ركن الأساس في بناء الأمة العربية هو اللغة العربية الفصحى التي تمتاز من بين لغات العالم الكبرى بتاريخها الطويل المتصل وثروتها الفكربية والأدبية، 
وحضارتها التي وصلت قديما الإنسانية بحديثها، وروابطتها التي لا تنقصم بكتاب

مقدس، ودين يزيد عن معتنقوه عن خمة سكان قي العالم. ."و اللغة العربية بمفهومها المعاصر هي اللغة الفصحى التى استخدمها العرب الجاهليون في أدبهم، وتلك اللغة التي نزل بها القرآن الكريم، والتي ما زالت تستخدم حتى اليوم في المواقف الخطاب

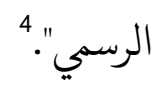
( ) خصائص اللغة العربية

من أهم الخصائص اللغة العربية أنها اللغة اشتقاقية، وهذا الاشتقاق أكسبها مرونة ومناعة في وقت واحد، فسمح لما بخلق ألفاظ جديدة، وحافظ على ثورتها، وحماها من الزيخ والشطط.

كذلك من خصائص اللغة العربية أنها في بنيتها وتركيبها لا تحتاج الجمل الخبربية فيها إلى إثبات ما يسمى في اللغات الأوربية "فعل الكينونة" وهو في الإنجليزية مثلا tobe فنحن نقول في العربية على سبيل الإخبار (فلان شجاع ) دون حاجة إلى

$$
\text { و أن نقول (فلان هوشجاع). }
$$

تعد اللغة العربية إحدى الوسائل المهمة في تحقيق المدرسة لوظائفها المتعددة،

لأن اللغة أهم وسائل الاتصال والتفاهم بين تلميذ وبيئته، وهي الأساس الذى تعتمد 4موسى حامد موسى خليفة،ملنحل إلى اللغويات، (الرياض: مكتبة الرشد-ناشرون،2009)،ص.139 
عليه تربيته من جميع النواحى، كما يعتمد عليه كل نشاط يقوم به سواء كان ذلك عن الاستماع والقراءة، أم عن طريق الكلام والكتابة.

ويهدف تعليم اللغة منذ بداية المرحلة الإبتدائية إلى تمكين الطفل من أدوات المعرفة عن طريق تزويده بالمهارات الأساسية في القراءة والكتابة والتعبير ومساعدته على اكتساب عاداتها الصحيحة واتجاهاته السليمة والتدرج في تنمية هذه المهارات على امتداد المراحل التعليمية بحيث يصل التلميذ في نهاية هذه المراحل إلى مستوى لغويّ يمكنه من استخدام اللغة استخداما صحيحا عن طريق التحدث، والكتابة، والقراءة، والاستماع، مما يساعده على أن ينهض بالعمل الذى يختاره، على أن يواصل الدراسة في المراحل التعليمية التالية.واللغة العربية ليست مادة لغوية فحسب لكنها وسيلة لدراسة المواد الأخرى التي تتعلق بالعلوم الشرعية، مثل :الفقه والحديث والتفسير والتوحيد وغير ذلك من العلوم الشرعية.

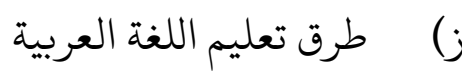
إن معرفة المدرس بطريقة التدريس وقدرته على استعمالها تساعد المدرس في معرفة الظروف التدريسية التي تحيط بالمواقف التعلمي، وهذه المعرفة تجعل عملية التعليم محببّة لدى المتعلم. 


\section{مفهوم الطريقة}

الطريقة في لغة هي المذهب، والسيرة، والمسلك، وجمعها الطرائق، قال تعالى " كنا

طرائق قددا (سورة الجن الأية :11). واصطلاحا الطريقة عبارة عن خطوات محددة يتبعها

المدرس لتحفيظ المتعلمين أكبر قدر من المادة التي تتصف بالجفاف والجحود. والطريقة

هي: مجموعة من الإجراءات لتحقيق أهداف متوخاة تشتمل على أنشطة تعليمية وتوظيف

كل مصادر التعلم المتاح وتعد وسيلة متقدمة للاتصال والتفاعل معه، وتعد مثيرا تعليما لسلوك المتعلم وتنظيم النشاط المعرفي له ووسيلة للابتكار والإبتداع. أهمية طرائق التدريس

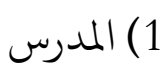
2) (المتعلم 3) المادة الدراسية ومن أنواع طريقة التدريس فيما يلي: 1 ) مريقة المحاضرة

من أقدم الطرائق التدريس وتسىى بالطريقة الإلقائية، حيث المدرس يلقي المرس والمتعلم مستمع إليه ، والمعلم يعد المادة للصغار كما يعد للكبار من دون التفكير في مستوى المتعلمين العقلي أو النظر إلى معارفهم.

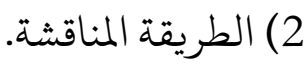


بأن يشارك ويتفاعل المتعلم مع المدرس في اكتساب المعارف والخبرات إذ يكون المتعلم والمدرس في موقيق إيجابي يتم من طريقة طرح الموضوع وتبادل الآراء المختلفة لدى المتعلمين ثم يعقب المدرس على ذلك بما هو صائب. 3) الطريقة الاستقرائية.

طريق الوصول إلى الأحكام العامة بواسطة الملاحظة والمشاهدة وبه يمكن الوصول إلى القضايا الكلية التي تسمى في العلوم باسم القوانين العلمية أو القوانين الطببعية. 4) - (4 الطريقة القياسية.

انتقال الفكر من الحهم على كلي إلى الحكم على جزئي أو جزئيات تحت هذا

الكي، وتعد الطريقة القياسية من أقدم الطرائق وفيها يتم الانتقال من القانون العام إلى القانون الخاصة ومن المبادئ العامة إلى النتائج. 5 ) طريقة التعلم التعاوني التعاون التعاوني: بأن يقسم طلبة الفصل على مجموعة صغيرة عدد أفراد المجموعة الواحدة ما بين (4-6) طلابا ويعطى كل مجموعة مهمة تعليمبية واحدة ويعمل كل مجموعة وفق الدور الذي كلف به وتعميم نتائج عمل المجموعة على المتعلمين.

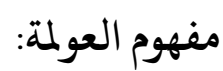
تعريف العولمة لغة: https://jurnal.yudharta.ac.id/v2/index.php/studi-arab 
العولمة لغة: مصدر الفعل "عَوْلَم" على صيغة "فوعل " (ويرى هذا الكلام أحمد

صدقي الدجاني)، و بعضهم يروا أن العولمة لعة هي: مشتقة من "عالم "و تجمع "عوامل " وكلمة العولمة نسبة إلى العالم، وتم تحليل لفظ العولمة لغوياً ليعني تعميم الشيء وتوسيع دائرته ليكتسب الصفة العالميّة، ويُعدّ لفظ العولمة لفظاً مترجماً للمصطلح الإنجليزي حيث ترجمه البعض بالعولمة، كما ترجمه البعض بالكونيّة، وورد أيضاً أنه (Globalization) تُرجم إلى لفظ الكوكبة، وقيل: العولمة هي الشوملة، ويُحدّ لفظ العولمة الأشهر بينها، فقد ). اشتُهِر بين الباحثين وأصبح الأكثر شيوعاً بين الاقتصاديّين، والسياسيّين، والإعلاميّين

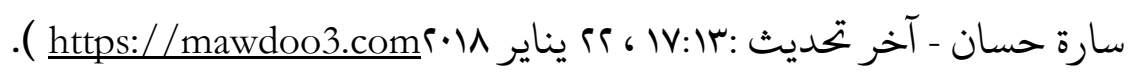
ب) تعريف العولمة اصطلاحا: هو توحيد جميع دول العالم على صبغة كاملة من ناحية المجال الاقتصاديّ، والاجتماعيّ، والفكريّ بغض النظر عن الجنسيّة، والعرق، والدين، والثقافة، وتمتدّ لتحكم وتضع روابط وقوانين وتزيح الحواجز المحدّدة بين الدول فيما بينها، وعرّفها صاحب، كتاب العولمة مالكوم واترز بأنها: "كل المستجدات والتطورات التي تسعى بقصد أو بدون قصد إلى دمج سكان العالم في مجتمع عالي واحد "، كما عرّفها المفكر رونالد روبرتسون البريطاني بأنّها : "اتجاه تاريخي نحو انكماش العالم وزيادة وعي الأفراد والمجتمعات بهذا الانكماش ."( سميحة ناصر - آخر تحديث :•ا:اع ، ع| يناير 19.؟ .h.htps://hyatok.com https://jurnal.yudharta.ac.id/v2/index.php/studi-arab 
144 | Volume 10, Nomor 2, Desember 2019

\section{استراتيجية تعليم اللغة العربية في عصر العولمة}

إن تدريس اللغة باتجاهه الحديث ينبنى أساسا على ما يسمى بفنون اللغة، وهي

أربعة : الاستماع، والحديث، والقراءة والكتابة .وتسمى هذه الفنون أحيانا "مهارة اللغة".وربما وراء اختيار كلمة "مهارة"هنا أن اللغة أساسا أدة الاتصال، والمهارة جزء أساسي وعامل مهم في دقة الاتصال ودقته وسرعته، كما أن اختيار كلمة "فن " تدل على أن الفرد حين يستخدم لغته يبتكر فيها ويبد ع.و أن تدريس اللغة العربية يمكن أن يكون أكثر فعالية إذا قام على أساس تناول"فنون اللغة "الاستماع، والقراءة، والكلام والكتابة، على أنها وحدات أساسية .من هنا يمكن أن نضع استراتيجية تعليم اللغة العربية في عصر العولمة على أساس فنون اللغة الأربع: الكلام، والكتابة، والقراءة والاستماع وبوسيلة الألعاب اللغوية.نظرالأهميتها وفعالياتها،ومن أهمية استراتيجية الألعاب هي: (1)إن اللعب أداة تربوية يساعد في إحداث تفاعل الفرد مع عناصر البيئة لغرض التعلم وإنماء الشخصية والسلوك (2)يمثل اللعب وسيلة تعليمية تقرب المفاهيم وتساعد في إدراك معانى الأشياء(3)يعتبر أداة فعالة في تفرد التعلم وتنظيمه لمواجهة الفروق الفردية وتعليم الأطفال وفقا لإمكاناتهم وقدراتهم.(4)يعتبر اللعب طريقة علاجية يلجأ إليها المربون لمساعدتهم في حل بعض المشكلات التي يعانى منها بعض الأطفال.(5)يشكل اللعب أداة تعبير وتواصل بين الأطفال.(6)تعمل الأطفال على تنشيط القدرات العقلية وتحسن الموهبة الإبداعية لدى

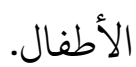


وإن الألعاب اللغوية تنقسم إلى أنواع متعددة فمن تقسيماتها تبعا للمهارات اللغوية الأساسيةوهي الألعاب الشفهية وألعاب القراءة والألعاب الكتابية،وعبرت بتريسيا أن الألعاب اللغوية تنقسم إلى خمسة أقسام حسب أهميتها الخاصة وهي الألعاب غير اللفظية (الألعاب التقدية (bord-advancing games)والألعاب اللفظية (nonverbal games) المركزة (word-focus games)والبحث عن الكنز (treasuer games)والألعاب التخديمية) (guessinggames)

وفي هذه وشة العمل ستبين الكاتبان عن الألعاب اللغوية المختلفة يمكن استعمالها في

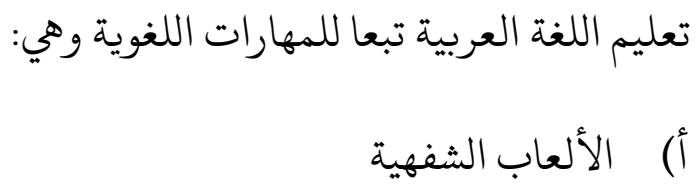
في تعليم اللغة يتعلم الناس باستعمال الألعاب وغالبا ما يكون التركيز على المشافهة في بدء برامج تعليم اللغة ويستمر النشاط الشفهي بعد ذلك حتى نهاية الدراسة. والاستفادة من الألعاب الشفهية في هذا المجال من تعليم اللغة بديل عن تكرار الممل وتخفيف من رتابة الدروس وتوفير لفرض عديدة للاستماع و الكلام في مواقف حية ومتعة تجعل الطلاب أكثر تفاعلا مع ما يدرسونه وأشد تجاوبا لهذا النوع من النشاط. فلا يمكننا أن نغفل ما للاستماع والكلامن دور رئيسي في إدارة الألعاب وإجرائها سواء من جانب

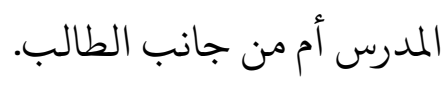

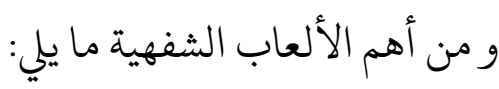
https://jurnal.yudharta.ac.id/v2/index.php/studi-arab 
146 | Volume 10, Nomor 2, Desember 2019

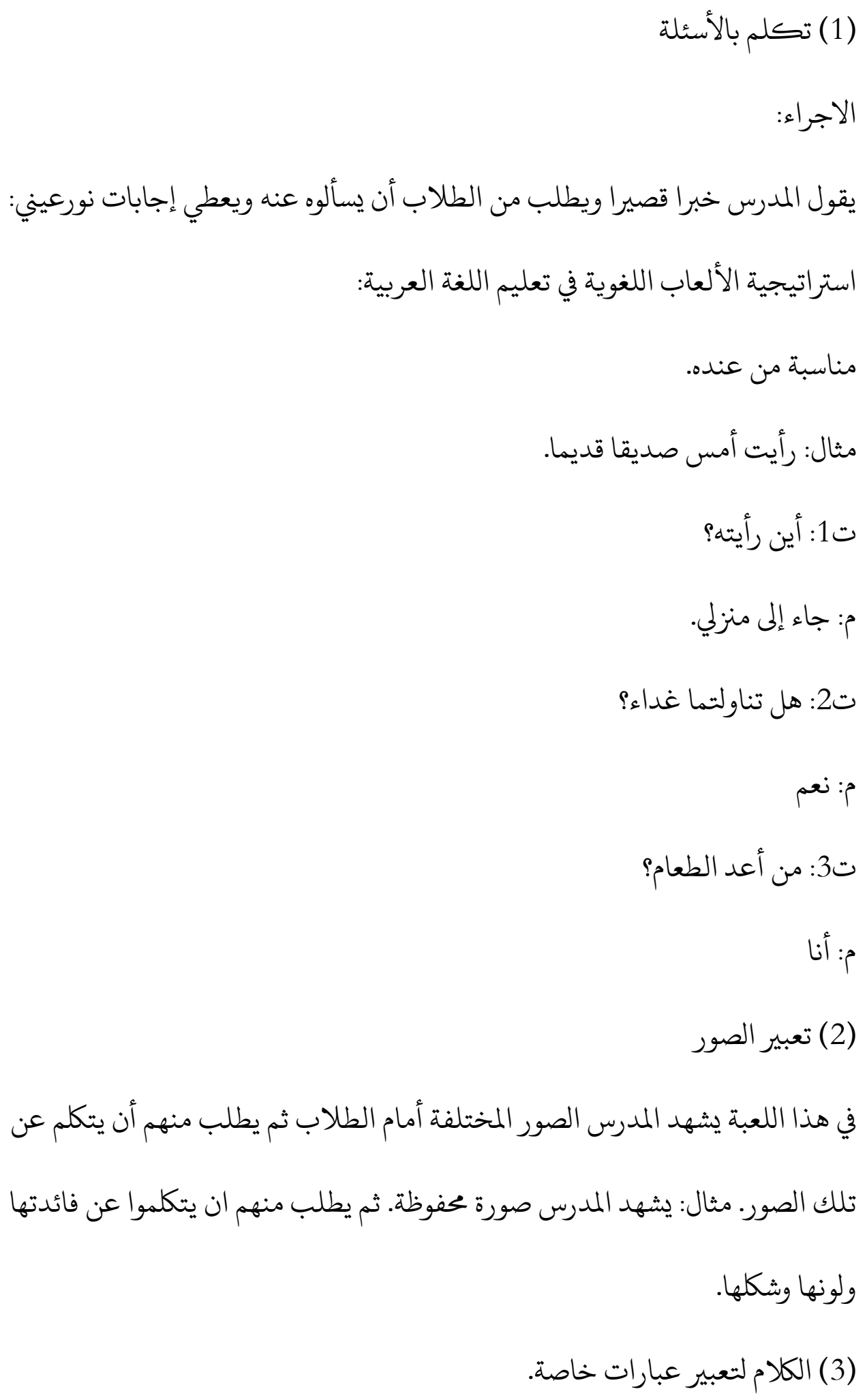

Program Studi Pendidikan Bahasa Arab Universitas Yudharta Pasuruan https:/jurnal.yudharta.ac.id/v2/index.php/studi-arab 
يقول المدرس العبارات خاصة بأن يقول جملة مفيدة من عبارات المدرس. مثال:

لا شك أن /طبعا/محا لا شك فيه/ بالتأكيد/ وفضلا عن ذلك/ وزيادة عن ذلك/ ومن ناحية

أخرى/ ومن جة أخرى. يواصل الطلاب مثلا: ممالاشك فيه أن المدرس ماهر.

$$
\text { ب) ألعاب القراءة }
$$

إن تعليم القراءة في أية لغة يعتبر إنجازا هاما بل اكتشاف جديد ـ هناك مشكلة لطلاب

الذين تعود القراءة من اليسار إلى اليمين أو من أعلى إلى أسفل لأن هذه الطريقة التي يقرؤون بها لغتهم الأم. إن الطلاب يحتاجون إلى ألعاب ما قبل القراءة حتى يدرك البعض المقصود بالقراءة ويدرب الآخرون حتى أسفل الصفحة.ويمكننا حصر أهم أنواع الألعاب التى تعالج مشكلات القراءة فيما يلي :(1)ألعاب التعرف على الحروف والكلمات والجملة(2)ألعاب التدريب على القراءة من اليمين إلى اليسار(3)ألعاب التدريب على قراءة كلمة أو عبارة ونص قصير(4)ألعاب التعرف على أخطاء القراءة وتصحيحها (5)ألعاب

$$
\text { وهناك ألعاب اخرى تستخدم في تنمية مهارة القراءة وهي: }
$$

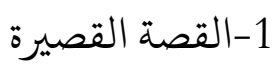

يقسم المدرس مجموعة قصص قصيرة إلى أربعة أجزاء.ويكتب كل جزء على بطاقة بلون واحد.يطلب من الطلاب الذين يحملون بطاقات خضراء مثلا أن يقرؤوا ما عليها واحد بعد آخر.ثم يشترك الطلاب في ترتيب أجزاء القصة .وبعد نجاحهم في ترتيب الصحيح 
يقرؤون القصة كاملة أمام كل الطلاب. ثم تبدأ مجموعة آخرى في قراءة أجزاء قصتها وترتيبها.

$$
\text { ج) الألعاب الكاتبية: الاعصة من الصورة }
$$

الإجراء: يقسم المدرس الطلاب إلى أربع فرق. ثم يأمر واحد من كل فرقة ليختار الصورة التي يحبها. ثم يأهر المدرس بأن يكتبوا القصة مناسبة في يصورة في أيديهم. وحدد المدرس الوقت حتى يسابق بعصهم بعضا في الكتابة. وحدد أيضا العناصر اللغة التي وجب على الطلاب أن يكتبوا في قصتهم. بعد الكتابة سأمر المدرس فرقة آخر بأن يصلح كتابة صديقه قبل أن يصححها المدرس.

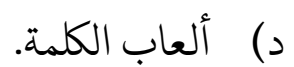
يعرض المدرس عدة الكمة غير مرتبة ويطلب المدرس من الطلاب أن يرتبوا ترتيبا سليما حتى تكون جملة مفيدة. مثلا: أ. قليل/بعد/المدرس/في المدرسة/وصل

يعرض المدرس على الطلاب عدة جمل غير مرتبة ويطلب المدرس من الطلاب أن يرتبوا الجمل حتى تكون فكرة واحدة. ثم يختار المدرس أحد الطلاب الذي يتقدم في القراءة

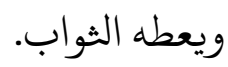
https://jurnal.yudharta.ac.id/v2/index.php/studi-arab 


\section{الخلاصة}

من هذا البحث وجد الباحثون استراتيجية المناسبة لتعليم اللغة العربية في عصر

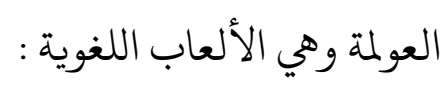

$$
\text { نظرا لأهميتها وفعالياتها،ومن أهمية استراتيجية الألعاب هي: }
$$

1) إن اللعب أداة تربوية يساعد في إحداث تفاعل الفرد مع عناصر البيئة لغرض

$$
\text { التعلم وإنماء الشخصية والسلوك. }
$$

2) يمثل اللعب وسيلة تعليمية تقرب المفاهيم وتساعد في إدراك معانى الأشياء

3) يعتبر أداة فعالة في تفرد التعلم وتنظيمه لمواجهة الفروق الفردية وتعليم الأطفال

$$
\text { وفقا لإمكاناتهم وقدراتهم. }
$$

4) يعتبر اللعب طريقة علاجية يلجأ إليها المربون لمساعدتهم في حل بعض المشكلات

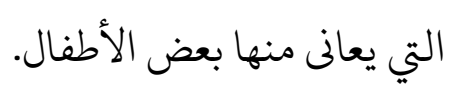

$$
\text { 5) يشكل اللعب أداة تعبير وتواصل بين الأطفال. }
$$

6) تعمل الأطفال على تنشيط القدرات العقلية وتحسن الموهبة الإبداعية لدى الأطفال. 
فتجي على يونس ومحمود كامل الناقة، 1977، أساسيات تعليم اللغة

$$
\text { العربية،(القاهرة:دار الثقافة للطباعة والنشر) }
$$

موسى حامد موسى خليفة، 2009، مدخل إلى اللغويات، (الرياض: مكتبة الرشد-

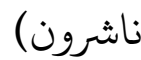

أبي الفتح ابني جني، 2001، الخصائص لابني جني، (بيروت:دار اكتب العلمية)

عبدالرحمن إبراهيم الفوزان، 1432، إضاءات لرفع كفاءة معلمي اللغة العربية

$$
\text { لغير الناطقين بها، (الرياض:فهرسية مكتبة الملك فهد الوطنية أثناء النشر) }
$$

عمران جاسم و حزة هاشم، 2013، مناهج و طرائق تدريس اللغة العربية، العراق

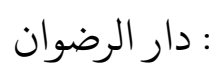

نور شمة، الألعاب اللغوية لتعليم اللغة العربية (النظرية العامة لاستخدام

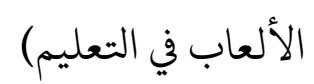

$$
\text { نورعيني، 2015، استراتيجية الألعاب في تعليم اللغة العربية، }
$$

